\title{
Unmutated and mutated chronic lymphocytic leukemias derive from self-reactive B cell precursors despite expressing different antibody reactivity
}

\author{
Maxime Hervé, ${ }^{1}$ Kai Xu, ${ }^{1}$ Yen-Shing Ng, ${ }^{1}$ Hedda Wardemann, ${ }^{2}$ Emilia Albesiano, ${ }^{3}$ \\ Bradley T. Messmer, ${ }^{3}$ Nicholas Chiorazzi, ${ }^{3}$ and Eric Meffre ${ }^{1,4}$ \\ ${ }^{1}$ Laboratory of Biochemistry and Molecular Immunology, Hospital for Special Surgery, New York, New York, USA. \\ 'Laboratory of Molecular Immunology, The Rockefeller University, New York, New York, USA. ${ }^{3}$ Institute for Medical Research, \\ North Shore - Long Island Jewish Health System and Departments of Medicine, North Shore University Hospital and \\ New York University School of Medicine, Manhasset, New York, USA. ${ }^{4}$ Weill Medical College of Cornell University, New York, New York, USA.
}

\begin{abstract}
B cell chronic lymphocytic leukemia (CLL) is a disease of expanding monoclonal B cells whose B cell receptor (BCR) mutational status defines 2 subgroups; patients with mutated BCRs have a more favorable prognosis than those with unmutated BCRs. CLL B cells express a restricted BCR repertoire including antibodies with quasi-identical complementarity-determining region 3 (CDR3), which suggests specific antigen recognition. The antigens recognized by CLL antibodies may include autoantigens since about half of CLL $B$ cells produce autoreactive antibodies. However, the distribution of autoreactive antibodies between $I g$ heavy-chain variable-unmutated ( $I g V$-unmutated) CLL (UM-CLL) and IgV-mutated CLL (M-CLL) is unknown. To determine the role of antibody reactivity and the impact of somatic hypermutation (SHM) on CLL antibody specificity, we cloned and expressed in vitro recombinant antibodies from M- and UM-CLL B cells and tested their reactivity by ELISA. We found that UM-CLL B cells expressed highly polyreactive antibodies whereas most M-CLL B cells did not. When mutated nonautoreactive CLL antibody sequences were reverted in vitro to their germline counterparts, they encoded polyreactive and autoreactive antibodies. We concluded that both UM-CLLs and M-CLLs originate from self-reactive B cell precursors and that SHM plays an important role in the development of the disease by altering original BCR autoreactivity.
\end{abstract}

\section{Introduction}

$\mathrm{V}(\mathrm{D}) \mathrm{J}$ recombination mediates $I g$ gene assembly during early B cell development and generates the naive B cell repertoire (reviewed in ref. 1, 2). During primary immune responses, naive B cells are activated through their B cell receptors (BCRs) and proliferate in germinal centers where their $I g$ genes undergo somatic hypermutation (SHM), thereby producing a new antibody repertoire (1). Little is known about the immunobiology of overexpanded B cell clones in chronic lymphocytic leukemia (CLL). The findings that SHM status of the Ig heavy-chain variable (IgV) genes can divide CLLs into 2 subgroups $(3,4)$ and that these subgroups experience markedly divergent clinical outcomes $(5,6)$ were important advances in the understanding of the disease. The presence of SHMs in the $\operatorname{IgV}$ genes of mutated CLLs (M-CLLs) correlates with a better prognosis than that of patients with $I g V$-unmutated CLLs (UM-CLLs) $(5,6)$. By analogy to normal B cell development, it has been postulated that UM-CLL $\mathrm{B}$ cells originate from a pregerminal center precursor with $I g$ genes devoid of somatic mutations whereas M-CLL B cells emerge from postgerminal center B cells that express BCRs altered by $\operatorname{SHM}(7,8)$. However, UM- and M-CLLs displayed similar gene expression pro-

Nonstandard abbreviations used: $\mathrm{BCR}$, B cell receptor; CDR3, complementaritydetermining region 3; CLL, chronic lymphocytic leukemia; IFA, immunofluorescence assay; IgV, Ig heavy-chain variable; M-CLL, IgV-mutated CLL; SHM, somatic hypermutation; UM-CLL, IgV-unmutated CLL.

Conflict of interest: The authors have declared that no conflict of interest exists.

Citation for this article: J. Clin. Invest. 115:1636-1643 (2005).

doi:10.1172/JCI24387. files, which suggests a common mechanism of transformation or cell origin $(9,10)$. The transformation events that select individual normal B cells to become CLL B cells remain unknown. CLL B cells express a distinct restricted antibody repertoire, which suggests a selection process driven by specific antigens (3, 4, 7, 8, 11-15). Moreover, the recent identification of quasi-identical BCRs expressed by different patients' CLL reinforces the idea that BCR reactivity may play an important role in the CLL transformation process (11-15). The antigens recognized by CLL antibodies remain to be identified, but they may include autoantigens since about half of CLL B cells have been reported to produce autoreactive antibodies $(16,17)$. However, the distribution of autoreactivity between UM- and M-CLL B cells is unknown. To determine the potential contribution of antibody reactivity to the differential progression of UM- and M-CLLs, we cloned and expressed in vitro antibodies from CLL B cells and tested their reactivity by ELISA and indirect immunofluorescence assays (IFAs) on HEp-2 cells (18-20). We found that most recombinant antibodies from UM-CLL B cells reacted with lysates of HEp-2 cells, DNA, insulin, and LPS and could therefore be considered polyreactive. In contrast, $M-C L L B$ cells expressed nonpolyreactive antibodies with or without HEp-2 reactivity. However, most nonreactive M-CLL antibodies that were reverted in vitro to germline sequences acquired a HEp-2 reactivity and/or polyreactivity similar to that of UM-CLL antibodies, which suggests that both M- and UM-CLL B cells derive from self-reactive $\mathrm{B}$ cell precursors. Moreover, differences in BCR reactivity between UM- and M-CLL may play a role in the differential development of the disease. 
A

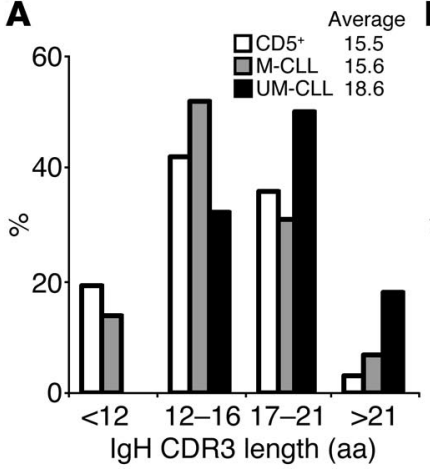

B

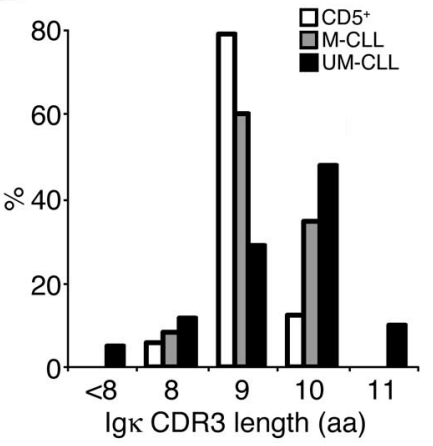

\section{Figure 1}

UM-CLL B cells express antibodies with long heavy- and light-chain CDR3s. $\operatorname{lgH}(\mathbf{A})$ and $\lg \kappa(B)$ CDR3 length in amino acids from control CD5+ (open bars), M-CLL (gray bars), and UM-CLL (black bars) B cells is indicated below the $x$ axes. The average CDR3 length for control $\mathrm{CD}^{+}, \mathrm{M}-\mathrm{CLL}$, and UM-CLL B cells was $15.5,15.6$, and 18.6 amino acids respectively. Significant $P$ values were obtained by Student's $t$ test when UM-CLL IgH CDR3 length was compared with that of control $\mathrm{CD}^{+}(P=0.004)$ and M-CLL $(P=0.008) \mathrm{B}$ cells. The $y$ axes indicate the frequency of $\lg \mathrm{H}$ and $\lg \kappa \mathrm{CDR} 3$ lengths in each group.

\section{Results}

$U M-C L L$ and some $M-C L L B$ cells express HEp-2 reactive antibodies. To determine the BCR reactivity of UM- and M-CLL B cells, we cloned and expressed in vitro recombinant antibodies from 57 cases of CLL (28 UM- and 29 M-CLLs) and compared them with 31 antibodies isolated from single $\mathrm{CD}^{+} \mathrm{B}$ cells from 3 healthy donors. We chose antibodies derived from this $\mathrm{B}$ cell subset as

controls because both UM- and M-CLL express the CD5 molecule and CD5-expressing $\mathrm{B}$ cells have been proposed as the precursors of CLL B cells $(21,22)$.

Consistent with previous reports $(3,4,11-15,23,24)$, we found that UM- and M-CLL B cells expressed a unique immunoglobulin repertoire that included specific $V_{H}, D$, and $J_{H}$ usage and sets of clones with quasi-identical complementarity-determining
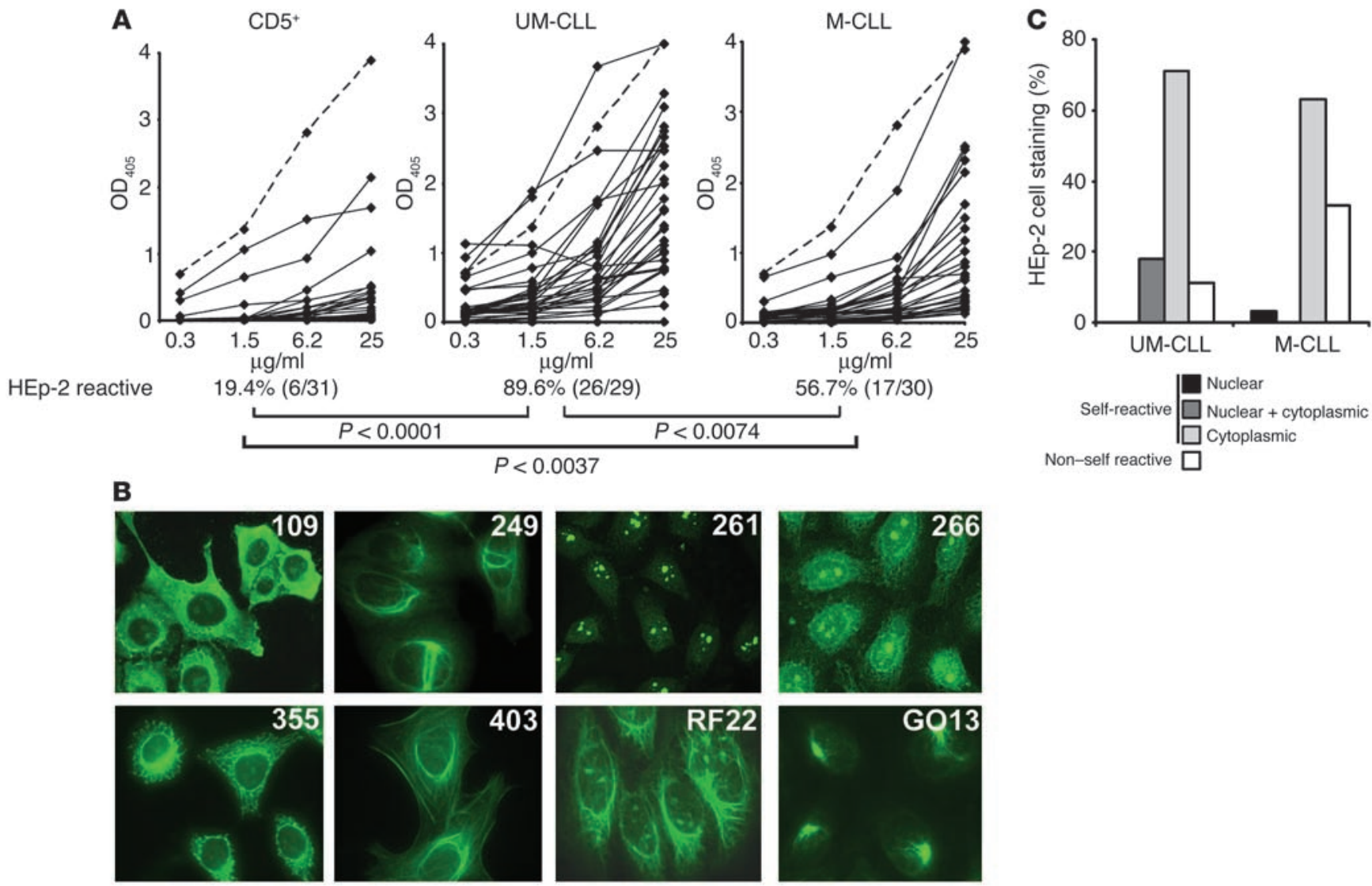

\section{Figure 2}

A majority of CLL B cells express HEp-2 reactive antibodies. (A) Data shown are from ELISAs for anti-HEp-2 cell reactivity of recombinant antibodies cloned from control CD5+ (left), UM-CLL (middle), and M-CLL (right) B cells. Dotted lines show ED38-positive control antibody (18, 19). The percentage of autoreactive clones for each fraction and their $P$ values are indicated. (B) CLL B cells mostly express anticytoplasmic antibodies. HEp-2-reactive antibodies from CLL B cells show various IFA patterns including rare nucleolar (CLL 261) and nuclear and cytoplasmic patterns (CLLs 266 and RF22) whereas most CLL antibodies recognized different structures in the cytoplasm (CLLs 249, 109, 403, 355, and GO13). (C) Frequency of self-reactive antibodies in UM-CLL (left) and M-CLL (right) with nuclear, nuclear/cytoplasmic, or cytoplasmic staining patterns and frequency of nonreactive antibodies. 
Table 1

Reactivity of highly similar antibodies from CLL patients

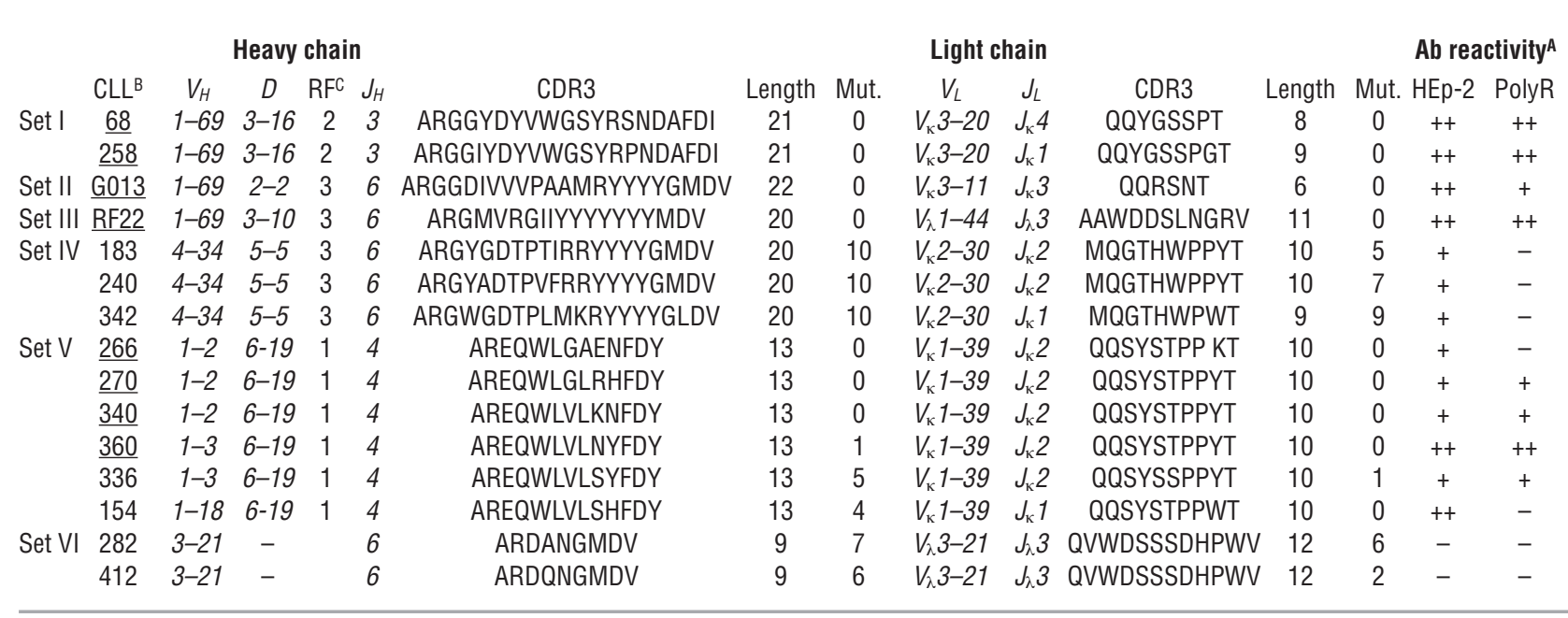

${ }^{A} \mathrm{Ab}$ reactivity was tested by $\mathrm{HEp}-2$ and polyreactivity (PolyR) ELISA. Abs were considered nonreactive when optical density at 405 nm (OD 405$)$ was inferior to $0.4(-)$ and reactive when $\mathrm{OD}_{405}$ was superior to either $0.4(+)$ or $1(++)$. BUM-CLLs are underlined. ${ }^{\mathrm{C}} \mathrm{RF}$, reading frame. The sets of CLL antibodies were previously reported (11-15). Mut., mutations.

region 3 (CDR3) (see Supplemental Figure S1 and Supplemental Table S1; supplemental material available online with this article; doi:10.1172/JCI24387DS1). Antibodies from UM-CLL were also remarkable in that they exhibited long heavy- and light-chain CDR3s (Figure 1, A and B). IgH CDR3s were significantly longer in UM-CLLs (18.6 aa on average) than in control $\mathrm{CD}^{+}$ (15.5 aa, $P=0.004)$ and M-CLL (15.6 aa, $P=0.008) \mathrm{B}$ cells (Figure 1A). UM-CLL $B$ cells also expressed longer IgK CDR3s than did control and M-CLL B cells, although differences failed to reach statistical significance (Figure 1B). Thus, UM-CLL B cells expressed an antibody repertoire that included clones with long $\operatorname{IgH}$ and Igא CDR3s.

The reactivity of recombinant antibodies was first tested against HEp-2 cells by ELISA and IFA, as previously reported $(18,19)$. We found that $19.4 \%(6 / 31)$ of the antibodies expressed by control $\mathrm{CD}^{+} \mathrm{B}$ cells were HEp-2-reactive antibodies and that this proportion was similar to the $20.4 \%$ of HEp-2-reactive antibodies found in mature naive $\mathrm{B}$ cells from control donors (Figure 2A) (18). In contrast, the proportion of HEp-2-reac-

\section{Figure 3}

CLL antibodies with quasi-identical Ig CDR3s recognize similar antigens. Antibodies belonging to 3 sets (sets I, IV, and V) of highly similar heavy and light chains as well as antibodies encoded by $\mathrm{V}_{\mathrm{H}} 1-69 / \mathrm{D} 3-3 / \mathrm{J}_{\mathrm{H}} 6$ show quasi-identical staining cytoplasmic patterns specific to each group. tive antibodies expressed by CLL B cells was significantly higher and represented $56.7 \%$ and $89.6 \%$ in M-CLL and UM-CLL cases, respectively (M-CLL vs. control $\mathrm{CD}^{+} \mathrm{B}$ cells, $P=0.0037$; UM-CLL vs. control $\mathrm{CD5}^{+} \mathrm{B}$ cells, $P<0.0001$ ) (Figure $2 \mathrm{~A}$ ). The higher
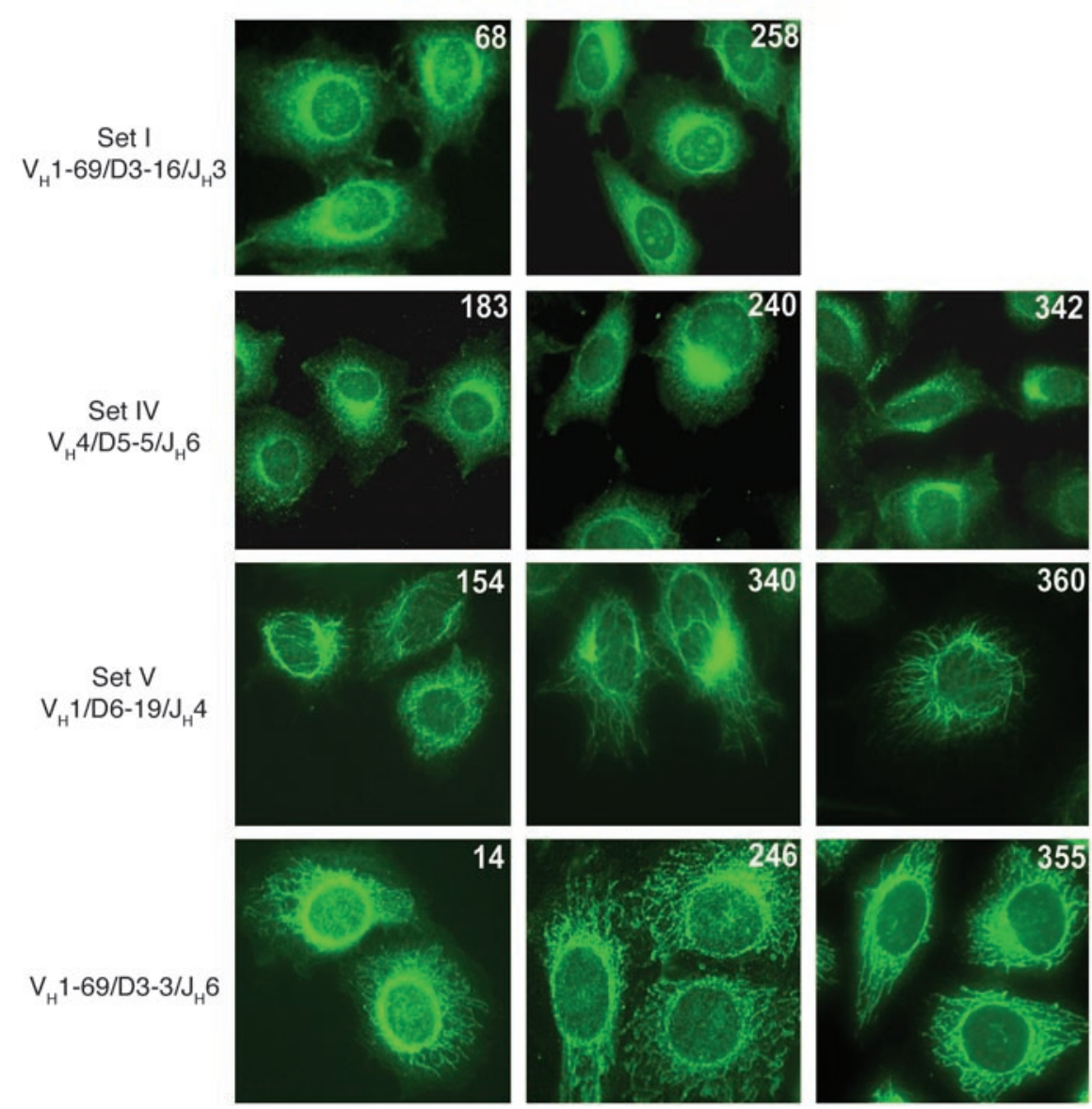

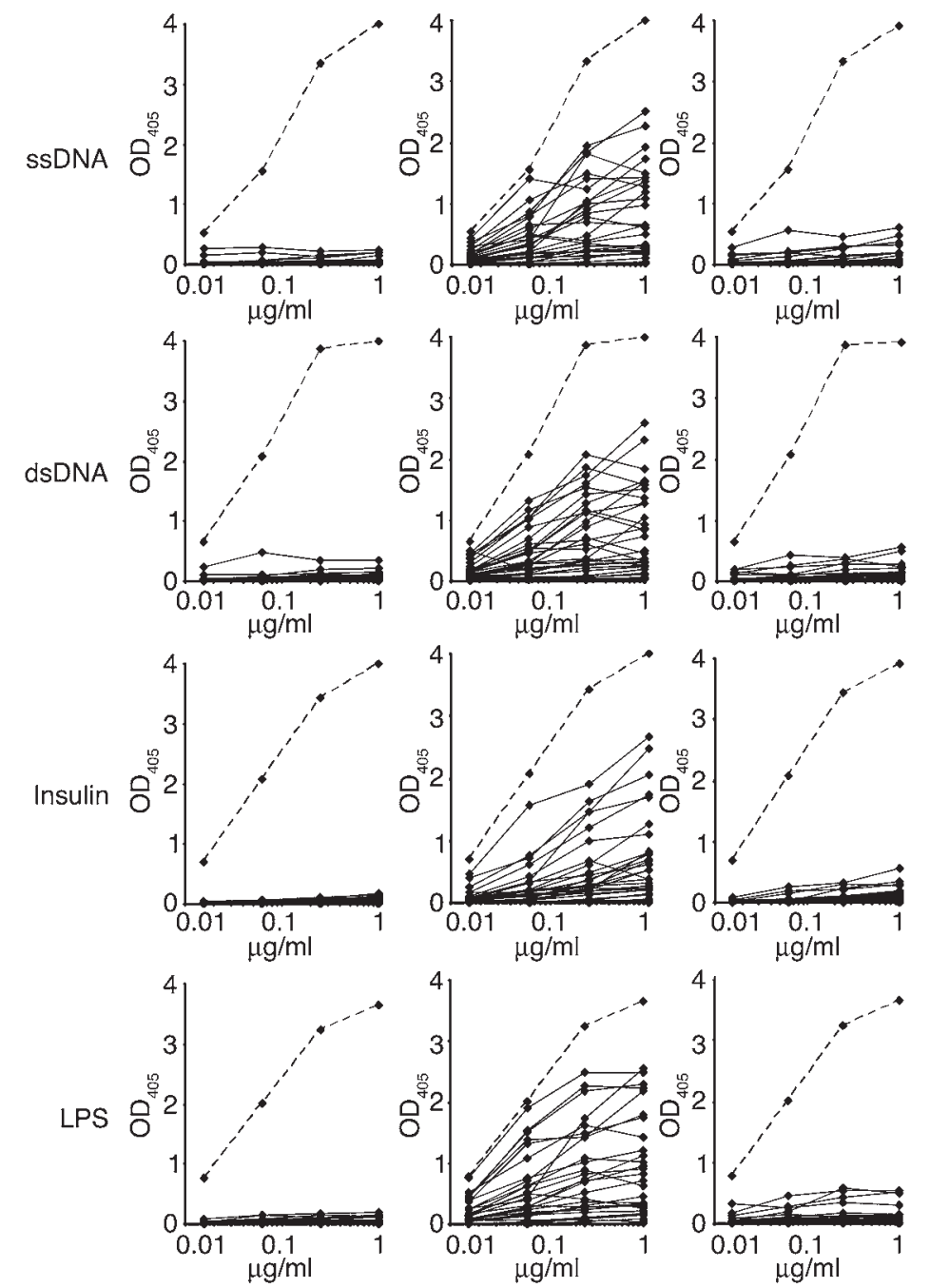

Polyreactive

$$
3.2 \%(1 / 31)
$$

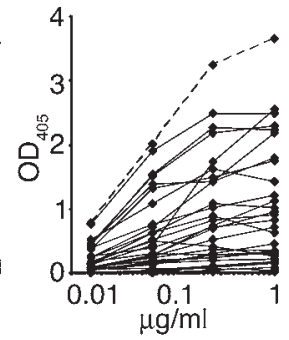

$79.3 \%(23 / 29)$

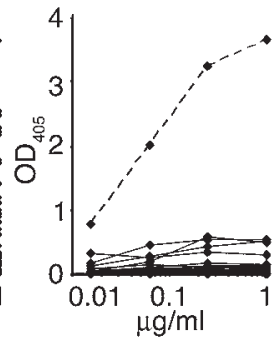

$13.3 \%(4 / 30)$

$P<0.0001$

$P<0.0001$

Figure 4

UM-CLL B cells express polyreactive antibodies. Antibodies cloned from control CD5+ (left), UM-CLL (middle), and M-CLL (right) B cells were tested by ELISAs for reactivity with ssDNA, dsDNA, insulin, and LPS. Dotted lines show ED38-positive controls $(18,19)$. Percentages represent frequency of polyreactive antibodies in each fraction. Significant $P$ values obtained when UM-CLL polyreactive frequency was compared with that of control $\mathrm{CD}^{+}$and $\mathrm{M}-\mathrm{CLL} \mathrm{B}$ cells are indicated.

incidence of HEp-2-reactive antibodies in UM-CLLs (89.6\%) compared with M-CLLs (56.7\%) was also statistically significant $(P=0.0074)$ (Figure $2 \mathrm{~A})$. Antibodies belonging to each CLL set showed a common reactivity: antibodies from sets I, II, III, IV, and $V$ were HEp-2 reactive whereas antibodies from set VI were not (Table 1). Most HEp-2-reactive antibodies from both UM- and M-CLLs recognized cytoplasmic structures whereas a minority reacted with nuclear components (Figure 2, B and C). Moreover, antibodies belonging to 3 previously described sets (sets I, IV, and V) (13-15) of quasi-identical heavy and light chains as well as antibodies encoded by $V_{H} 1-69 / D 3-3 / J_{H} 6$ displayed very similar IFA patterns, suggesting that the mAb of each group may recognize the same cytoplasmic structures
(Figure 3). We concluded that about half of the antibodies expressed by M-CLLs are HEp-2 reactive whereas most of UM-CLLs express HEp-2-reactive antibodies that recognize cytoplasmic antigens. In addition, the cytoplasmic structures recognized by the quasiidentical mAbs from different patients with CLL are restricted and similar, which suggests that CLL antibodies that belong to one set may recognize the same antigens and a limited number of epitopes.

$U M-C L L$ but not $M$-CLL B cells express polyreactive antibodies. About half of CLL B cells have been reported to express polyreactive antibodies $(16,17)$. However, the extent to which polyreactivity is distributed between UM- and M-CLLs is unknown. To investigate this distribution and the impact of somatic mutation on the multireactivity of CLL antibodies, we used ELISA to test the binding of UM- and M-CLL antibodies to a panel of specific antigens $(18,19)$. We previously reported that only $4.3 \%$ of mature naive B cells expressed antibodies with low levels of polyreactivity (18). We found that control $\mathrm{CD}^{+} \mathrm{B}$ cells expressed a similar proportion $(3.2 \%, 1 / 31)$ of polyreactive antibodies as did control mature naive B cells (Figure 4). M-CLLs expressed a slightly increased frequency $(13.3 \%, 4 / 30)$ of polyreactive antibodies that did not reach statistical significance (M-CLL vs. control CD5 ${ }^{+} \mathrm{B}$ cells; $P=0.20$ ) (Figure 4). In contrast, $79.3 \%$ of UM-CLL antibodies were polyreactive (UM-CLL vs. control $\mathrm{CD5}^{+} \mathrm{B}$ cells, $P<0.0001$; UM-CLL vs. M-CLL, $P<0.0001)$. Antibodies from UM-CLLs also showed high levels of polyreactivity when compared with those from control $\mathrm{CD}^{+} \mathrm{B}$ cells and M-CLLs (Figure 4). In addition, UM-CLL antibodies from sets I, II, and III were polyreactive whereas antibodies from M-CLL sets IV and VI were not (Table 1). Antibodies from CLL group V showed a more diverse reactivity, and only some of these antibodies were polyreactive, further demonstrating that this large set of CLLs that regroups UM- and M-CLL cases is heterogeneous (Table 1). Thus, most UM-CLLs express highly polyreactive antibodies whereas control $\mathrm{CD}^{+} \mathrm{B}$ cells and M-CLLs do not.

Nonautoreactive $M-C L L$ antibodies derive from unmutated polyreactive/HEp-2-reactive antibodies. SHM plays a major role in the development of mature B cells by altering $\mathrm{BCR}$ reactivity. To determine whether nonautoreactive M-CLL antibodies derived from polyreactive antibodies that were altered by SHM (25), we reverted in vitro M-CLL antibody heavy- and light-chain genes to their original unmutated sequences. Because heavy-chain CDR3s play an essential role in conferring antibody polyreactivity (26), we selected for reversion M-CLL antibodies with mutated $D$ and $J_{H}$ gene segment sequences that we could be assured were assignable to known germline counterparts in order to revert CDR3 sequences as reliably as possible (Figure 5, A and B). We tested the binding of reverted antibodies (revertants) by ELISA on HEp-2 cell extracts and on a panel of specific antigens and compared their reactivity with their mutated counterparts (Figure 6, A and B). Out of 8 revertants, 6 (183R, 240R, 342R, 374R, 189R, and 215R) showed increased HEp-2 reactivity and/or acquired polyreactivity (Figure 6, A and B). Most revertants showed different IFA- 
A

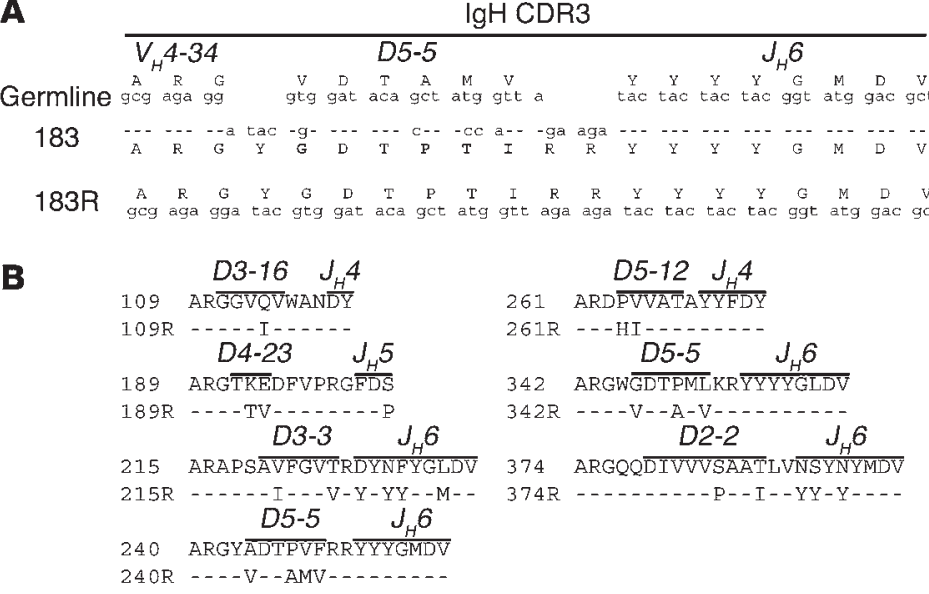

\section{Figure 5}

IgH CDR3 sequences of unmutated revertant antibodies engineered from M-CLL antibodies. (A) The 183 mutated sequence (183) to be reverted according to germline $V_{H}, D$, and $J_{H}$ counterparts was selected based on the identification of mutations in bold in the IgH CDR3 sequences. Homologous nucleotides to the original germline segment sequences are represented by dots whereas nucleotides to be substituted in the reverted sequence are shown in bold. Deducted amino acids are shown in upper-case letters. 183R, the resulting sequence of the 183 unmutated revertant. (B) IgH amino acid CDR3 sequences of mutated-CLL and engineered unmutated revertants $(\mathrm{R})$. Homologous amino acids to the original mutated CLL sequences (top) are represented by dots whereas substituted amino acids in the reverted sequence (bottom) are shown. IgH CDR3 $D$ and $J_{H}$ gene segments are indicated. staining patterns when compared with their mutated counterparts (Figure 6C). For instance, $342 \mathrm{R}$ reacts with the cytoplasm and the nucleus whereas its mutated counterpart only binds cytoplasmic structures (Figure 6C). In addition, the 2 rever- tants that did not show an increase in HEp-2 cell reactivity and polyreactivity, 109R and 261R, displayed different IFA-staining patterns compared with their mutated counterparts. For example, $261 \mathrm{R}$ stained the cytoplasm whereas the original mutated

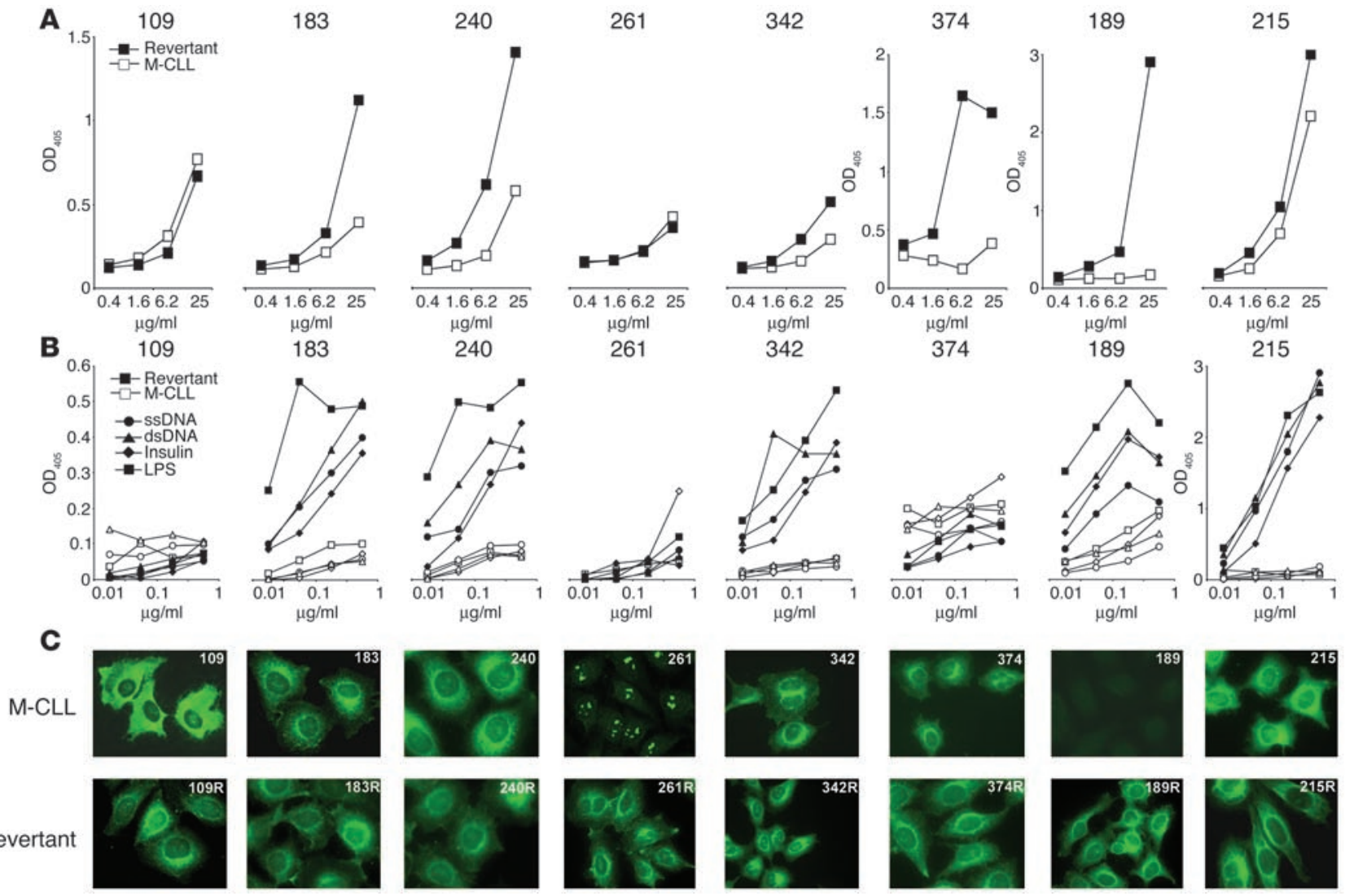

\section{Figure 6}

Most unmutated revertant antibodies engineered from M-CLL antibodies are HEp-2 reactive and/or polyreactive. (A) HEp-2 reactivity is increased in revertant CLL antibodies. Data shown are from ELISAs for anti-HEp-2 cell reactivity of revertant (filled squares) and M-CLL antibodies (open squares). (B) A majority of revertant CLL antibodies acquire polyreactivity. Revertant (filled symbols) and M-CLL antibodies (open symbols) were tested by ELISAs for reactivity with ssDNA (circles), dsDNA (triangles), insulin (diamonds), and LPS (squares). (C) Revertant CLL antibodies show IFA staining patterns that are different from those of their M-CLL counterparts. Mutated CLL HEp-2 staining patterns (top) were compared with unmutated revertant patterns (bottom). CLL 261 reverts from nucleolar (CLL 261) to cytoplasmic (CLL 261R) whereas others modify (CLLs 109R, 183R, 240R, 342R, 374R, and 215R) or acquire (CLL 189R) cytoplasmic staining patterns. 


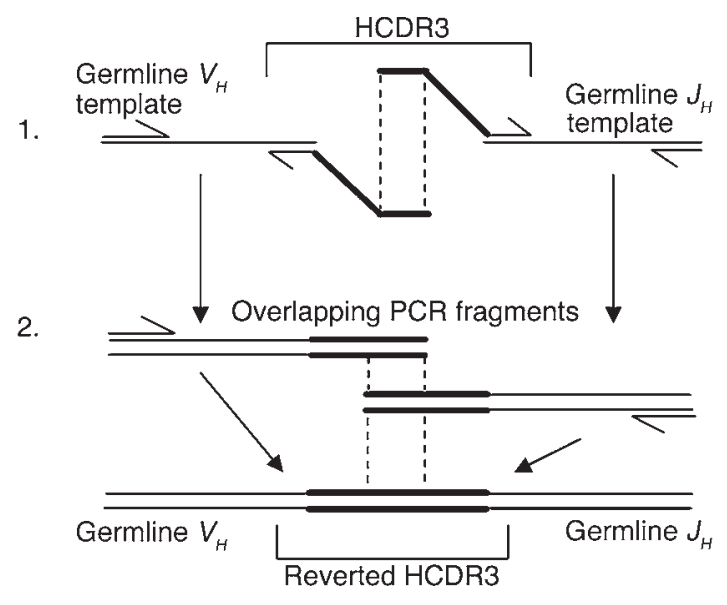

Figure 7

Strategy to revert mutated CLL antibodies to unmutated original counterpart. (1) Using a multi-template PCR strategy, germline $V_{H}$ and $J_{H}$ templates were first amplified with overlapping reverted CDR3 primers combined with primers annealing with either upstream germline $V_{H}$ genes or downstream $J_{H}$ segments. (2) A second PCR reaction fused the 2 overlapping $V_{H}$ and $J_{H}$ PCR fragments generating germline revertant antibody seqence.

261 antibody exhibited an antinuclear pattern (Figure 6C). We concluded that in all (8/8) CLL clones studied, SHM altered the original autoreactivity of antibodies produced by M-CLL cases. Moreover, when somatic mutations were replaced with the original amino acids, most revertants showed HEp-2 staining patterns that were different from those of their mutated counterparts, which revealed that SHM reshaped their autoreactivity toward different antigens.

\section{Discussion}

A role for BCRs in the development of CLL B cells was first suggested by the expression of a restricted antibody repertoire by both UM- and M-CLL B cells $(3,4,23,24)$. The idea of CLL B cell selection by specific antigens/autoantigens was further reinforced by the identification of highly similar $\mathrm{V}(\mathrm{D}) \mathrm{J}$ rearrangements in different patients. Sets of quasi-identical antibodies have been identified in both UM- and M-CLL, which suggests that selection by a limited number of antigens occurred in both groups (11-13). Our data refine these observations by suggesting that B cells that eventually transform into CLL B cells of both the mutated and the unmutated subgroups of patients originally derive from autoreactive clones. However, the nature of the (auto-)antigens that may be involved in the CLL transformation process remains to be determined.

Immunofluorescence stainings of HEp-2 cells revealed that most UM- and M-CLL antibodies recognize diverse cytoplasmic structures, which suggests that several antigens could favor and/or support the development of CLL B cells. However, UM- and M-CLL antibodies that belong to the same sets of quasi-identical heavy- and light-chain CDR3s showed similar staining patterns, which suggests that the same cytoplasmic antigens supported the development of those quasi-identical B cell clones in different patients (11-13). Most CLLs expressing mutated antibodies reacted with cytoplasmic epitopes that were different from those recognized by the germline counterparts from which they originated, revealing that SHM shaped M-CLL $\mathrm{BCR}$ reactivity toward some specific new antigens.

Based on the mutational status of antibodies expressed by CLL cases, it is believed that UM-CLLs originate from pregerminal center B cell precursors whereas M-CLLs derive from postgerminal center B cells that have been affected by SHM (7). However, UM- and M-CLL B cells showed similar gene expression profiles that argued for either a common B cell origin or mechanism of transformation $(9,10)$. BCR reactivity from UMand M-CLL B cells can help in tracing the origin of those cells. We found that UM-CLL B cells expressed highly polyreactive antibodies whereas most M-CLL B cells did not. We further demonstrated that mutated nonautoreactive CLL antibodies originate from polyreactive and/or autoreactive antibodies similar to those from UM-CLLs. It is therefore likely that both Mand UM-CLL B cells derive from B cells that have in common the expression of autoreactive BCRs. However, which specific $B$ cell subset(s) represents the CLL precursor population(s) is still unclear. $\mathrm{CD}^{+} \mathrm{B}$ cells have been proposed as the precursors of CLL B cells because UM- and M-CLL B cells also express CD5 and because both $\mathrm{CD}^{+}$and CLL B cells have been reported as expressing polyreactive antibodies $(21,22,27,28)$. However, this hypothesis was not supported by gene-expression profiling studies that compared peripheral $\mathrm{CD5}^{+} \mathrm{B}$ cells with UM- and M-CLL B cells $(9,10)$. Consistent with these data, we found that antibodies from $\mathrm{CD}^{+} \mathrm{B}$ cells did not express polyreactive antibodies whereas UM-CLL B cells did. The apparent discrepancy from previous reports indicating that $\mathrm{CD} 5^{+} \mathrm{B}$ cells express autoreactive antibodies $(27,28)$ may reside in the different methods used to analyze antibody reactivity. Our RT-PCR-based method produces recombinant monovalent IgG antibodies whereas Epstein Barr virus-transformed B cells may secrete multivalent pentameric IgMs whose avidity favors increased levels of autoreactivity. Nonetheless, our data demonstrate that UM-CLL B cells express antibodies with higher levels of polyreactivity than those of $\mathrm{CD}^{+} \mathrm{B}$ cells, suggesting that these cells might not be the precursors of CLL B cells. Therefore, CLLs could derive from another $\mathrm{B}$ cell population that expresses autoreactive antibodies such as $\mathrm{V}$-preB $\mathrm{B}^{+} \mathrm{L}^{+} \mathrm{B}$ cells (19). $\mathrm{V}$-preB ${ }^{+} \mathrm{L}^{+} \mathrm{B}$ cells are a small population of peripheral $B$ cells that coexpress surrogate and conventional light chains and display a unique antibody repertoire that is reminiscent of some UM-CLLs $(29,30)$. In particular, $V_{H} 1-69$ antibodies display long CDR3s, biased $J_{H} 6$ usage, and a preference for hydrophobic $D$ reading frame in both $\mathrm{V}$-preB ${ }^{+} \mathrm{L}^{+}$and UM-CLL B cells (sets II and III, Table 1; refs. $13,30,31)$. In addition, $V$-preB ${ }^{+} L^{+} B$ cells and $U M$-CLLs express HEp-2-reactive and polyreactive antibodies, further supporting this hypothesis (19). Alternatively, CLL cells may emanate from human counterparts of mouse B-1 and marginal zone B cells $(7,32)$. Each of these $B$ cell subpopulations can, like $V$ preB ${ }^{+} L^{+} B$ cells, express autoreactive BCRs with recurrent $V(D) J$ combinations, and marginal zone $\mathrm{B}$ cells in mice can have BCR restrictions that resemble those in CLL B cells $(19,33,34)$. However, the antibody reactivity of human marginal zone B cells that have been reported to have a phenotype similar to that of blood-circulating $\mathrm{CD} 19^{+} \operatorname{IgM}{ }^{+} \operatorname{IgD}^{+} \mathrm{CD} 27^{+} \mathrm{CD} 1 \mathrm{c}^{+} \mathrm{B}$ cells $(35)$ remains to be determined before those cells can be considered as potential CLL precursors.

An apparent contradiction to the hypothesis of a common origin of UM- and M-CLL B cells resides in their different anti- 
body repertoires. UM- and M-CLL B cells usually express different $V_{H}$ genes $(4,24)$. In addition, the repertoire of UM-CLL is remarkable in that it is enriched in clones with long heavyand light-chain CDR3s $(4,24)$ that favor self reactivity $(18,19)$. However, both UM- and M-CLL B cells show an increase in $J_{H} 6$ usage and display sets of quasi-identical antibodies (4, 11-15). UM- and M-CLL B cells may derive from an autoreactive B cell population that may contain recurrent $\mathrm{B}$ cell clones in healthy individuals. A restricted set of (auto-)antigens may be able to trigger the amplification of some specific clones that may have acquired growth advantage through an initial DNA lesion. The different nature of those antigens may induce SHMs in some clones but not in others, thereby segregating CLL precursors into mutated and unmutated cases according to the initial structure of their antigen receptors.

Could BCR autoreactivity that is altered by SHM be responsible for the different clinical courses followed by UM- and M-CLL cases? Antibodies expressed by UM-CLL B cells are highly polyreactive whereas almost none of the M-CLL antibodies are. Self and foreign antigens may repetitively trigger UM-CLL B cells $(7,11)$ and generate intracellular signals potentially mediated by ZAP70 and Syk (36-38). Consistent with this hypothesis, the capacity of the BCR to transduce intracellular signals is retained in UM-CLL compared with anergic M-CLL (11, 36-39). UM-CLL B cells have shorter telomeres than M-CLLs, which suggests an increased proliferative history in UM-CLLs compared with that of M-CLLs (40). In a recent study, a high CLL birth rate correlated with a higher risk for patients developing a progressive disease (41). The reactivity of many of the antibodies from those patients was analyzed in our report. Most CLL patients with high birth rates expressed polyreactive antibodies whereas those with lower birth rates expressed nonpolyreactive antibodies (41). Thus, through recurrent BCRmediated signaling, polyreactivity may play a role in accelerating the development of the disease.

\section{Methods}

CLL patients. 57 patients with expansions of $\mathrm{CD}^{+} \mathrm{CD} 19^{+} \mathrm{B}$ cells expressing diminished levels of surface membrane IgM or IgG were chosen from a cohort of 237 patients with clinical and laboratory features of CLL. Most of them were randomly chosen whereas patients with CLL that expressed antibodies with quasi-identical CDR3s were selected to be included in the study (11-15). IgV gene sequences from some patients were described previously $(4,12,13)$. Based on the mutational status of $\operatorname{Ig} V_{H}$ genes, the cohort included 28 patients with unmutated $\operatorname{Ig} V_{H}$ genes (< $2 \%$ mutations at the DNA level) and 29 patients with mutated $\operatorname{Ig} V_{H}$ gene sequences ( $>2 \%$ mutations). All samples were collected after patients signed informed consent in accordance with protocols reviewed by the Institutional Review Board of North Shore University Hospital and Long Island Jewish Medical Center.

Single-cell sorting. Peripheral B cells were purified from the blood of CLL patients and 3 unrelated healthy donors by negative selection using RosetteSep procedure (StemCell Technologies). Peripheral $\mathrm{CD}^{+} \mathrm{B}$ cells from control donors were further enriched by positive selection with anti-PE magnetic microbeads (Miltenyi Biotec) after PE anti-human CD5 staining (BD Biosciences - Pharmingen). CD5-enriched peripheral B cells from control donors were stained with anti-human IgM biotin and allophycocyanin anti-human CD19 (BD Biosciences - Pharmingen). Biotinylated antibodies were revealed using PE-cy7 conjugated streptavidin (BD Biosciences - Pharmingen). Single $\mathrm{CD} 19^{+} \mathrm{CD}^{+} \mathrm{IgM}^{+} \mathrm{B}$ cells from control donors were sorted on a FACSVantage SE (BD) into 96-well PCR plates containing $4 \mu \mathrm{l}$ Lysis solution $(0.5 \times$ PBS containing $10 \mathrm{mM}$ DTT, 8U RNAsin, Promega; 0.4U 5'-3' RNAse inhibitor, Eppendorf) and were immediately frozen on dry ice. All samples were stored at $-70^{\circ} \mathrm{C}$.

cDNA, PCRs, antibody production and purification. Total RNA was extracted from $10^{4}-10^{5}$ enriched CLL B cells using TRIzol Reagent (Gibco-BRL, Invitrogen Corp.). RNA was reverse-transcribed in $10 \mu \mathrm{l} 1 \times$ reaction buffer (150 ng random hexamer primer N6 [Amersham Pharmacia Biotech Inc.], $10 \mathrm{mM}$ dNTP [Roche], 0.1 M DTT, 0.5\% NP40, 4U RNAsin [Promega], and 6U Prime RNAse Inhibitor [Eppendorf]) with Superscript II (Gibco-BRL, Invitrogen Corp.), and RT-PCR reactions were performed using primers to amplify I $g$ genes that include restriction sites compatible with expression vectors previously described (18). RNA from single cells was reverse-transcribed in the original 96-well plate in $12.5 \mu 1$ $1 \times$ reaction buffer containing $100 \mathrm{U}$ of Superscript II RT (Gibco-BRL, Invitrogen Corp.) for 45 minutes at $37^{\circ} \mathrm{C}$. RT-PCR reactions, primer sequences, cloning strategy, expression vectors, antibody expression, and purification were as previously described (18). Ig sequences were analyzed by Ig BLAST comparison (http://www.ncbi.nlm.nih.gov/projects/igblast/) with GenBank.

Strategy to revert mutated CLL antibodies to unmutated original counterparts. Using a multitemplate PCR strategy, we first amplified germline $V_{H}$ and $J_{H}$ templates with reverted CDR3 primers consisting of overlapping germline $D_{H}$ and/or $J_{H}$ sequences combined with primers annealing with either upstream germline $V_{H}$ genes or downstream $J_{H}$ segments (see Figure 7 and Supplemental Table S4). A 20-cycle second PCR reaction fused the 2 overlapping $V_{H}$ and $J_{H}$ PCR fragments, generating germline revertant antibody sequences (Figure 7). Because light-chain genes show limited $V$-J-junction diversity, we were able to find germline counterparts with identical $V-J$ junction in the 395 light-chain genes cloned in previous studies (18-20).

ELISAs and IFAs. Antibody concentrations, reactivity against specific antigens, and indirect immunofluorescence were as described (18). High (polyreactive ED38) and weak (mGO186) HEp2-reactive and nonreactive mGO53 were used as positive and negative controls in HEp-2 reactivity and polyreactivity ELISAs $(18,19)$.

Statistical analysis. $P$ values were calculated by 2 -tailed Fisher exact test. Student's $t$ test was used to analyze CDR3 length. Probability values of $P<0.05$ were considered to be statistically significant.

Supplementary material. Antibody repertoires from control $\mathrm{CD}^{+}$as well as UM- and M-CLL B cells are analyzed in Supplemental Figure S1 $\left(V_{H}, \mathrm{D}\right.$, $J_{H}$ usage). Antibody repertoires from UM- and M-CLL as well as control $\mathrm{CD}^{+} \mathrm{B}$ cells are presented in Supplemental Tables S1, S2, and S3. Primers to revert M-CLL antibodies are described in Supplemental Table S4.

\section{Acknowledgments}

We thank K. Velinzon for help with the sorting of single cells and J. Inra for technical assistance. This work was supported by a grant from the Dana Foundation (to E. Meffre) and by R01 grants from the National Cancer Institute (CA 81554 and CA 87956) and a General Clinical Research Center Grant (M01 RR018535) from the National Center for Research Resources (to N. Chiorazzi).

Received for publication January 4, 2005, and accepted in revised form March 23, 2005.

Address correspondence to: Eric Meffre, Hospital for Special Surgery, Research Department, 535 East 70th Street, New York, New York 10021, USA. Phone: (212) 774-2347; Fax: (212) 717-1192; E-mail: meffree@hss.edu. 
1. Rajewsky, K. 1996. Clonal selection and learning in the antibody system. Nature. 381:751-758.

2. Meffre, E., Casellas, R., and Nussenzweig, M.C. 2000. Antibody regulation of B cell development. Nat. Immunol. 1:379-385.

3. Schroeder, H.W., Jr., and Dighiero, G. 1994. The pathogenesis of chronic lymphocytic leukemia: analysis of the antibody repertoire [review]. Immunol. Today. 15:288-294.

4. Fais, F., et al. 1998. Chronic lymphocytic leukemia B cells express restricted sets of mutated and unmutated antigen receptors. J. Clin. Invest. 102:1515-1525.

5. Damle, R.N., et al. 1999. Ig V gene mutation status and CD38 expression as novel prognostic indicators in chronic lymphocytic leukemia. Blood. 94:1840-1847.

6. Hamblin, T.J., Davis, Z., Gardiner, A., Oscier, D.G., and Stevenson, F.K. 1999. Unmutated Ig V(H) genes are associated with a more aggressive form of chronic lymphocytic leukemia. Blood. 94:1848-1854.

7. Chiorazzi, N., and Ferrarini, M. 2003. B cell chronic lymphocytic leukemia: lessons learned from studies of the B cell antigen receptor. Annu. Rev. Immu nol. 21:841-894.

8. Stevenson, F.K., and Caligaris-Cappio, F. 2004. Chronic lymphocytic leukemia: revelations from the B-cell receptor. Blood. 103:4389-4395.

9. Rosenwald, A., et al. 2001. Relation of gene expression phenotype to immunoglobulin mutation genotype in B cell chronic lymphocytic leukemia. J. Exp. Med. 194:1639-1647.

10. Klein, U., et al. 2001. Gene expression profiling of B cell chronic lymphocytic leukemia reveals a homogeneous phenotype related to memory B cells. J. Exp. Med. 194:1625-1638.

11. Tobin, G., Soderberg, O., Thunberg, U., and Rosenquist, R. 2004. V(H)3-21 gene usage in chronic lymphocytic leukemia-characterization of a new subgroup with distinct molecular features and poor survival [review]. Leuk. Lymphoma. 45:221-228.

12. Ghiotto, F., et al. 2004. Remarkably similar antigen receptors among a subset of patients with chronic lymphocytic leukemia. J. Clin. Invest. 113:1008-1016. doi:10.1172/JCI200419399.

13. Messmer, B.T., et al. 2004. Multiple distinct sets of stereotyped antigen receptors indicate a role for antigen in promoting chronic lymphocytic leukemia. J. Exp. Med. 200:519-525.

14. Widhopf, I.G., et al. 2004. Chronic lymphocytic leukemia B cells of over one percent of patients express virtually identical immunoglobulins.
Blood. 104:2499-2504.

15. Tobin, G., et al. 2004. Subsets with restricted immunoglobulin gene rearrangement features indicate a role for antigen selection in the development of chronic lymphocytic leukemia. Blood. 104:2879-2885.

16. Sthoeger, Z.M., et al. 1989. Production of autoantibodies by CD5-expressing B lymphocytes from patients with chronic lymphocytic leukemia. J. Exp. Med. 169:255-268.

17. Borche, L., Lim, A., Binet, J.L., and Dighiero, G. 1990. Evidence that chronic lymphocytic leukemia B lymphocytes are frequently committed to production of natural autoantibodies. Blood. 76:562-569.

18. Wardemann, H., et al. 2003. Predominant autoantibody production by early human B cell precursors. Science. 301:1374-1377.

19. Meffre, E., et al. 2004. Surrogate light chain expressing human peripheral B cells produce self-reactive antibodies. J. Exp. Med. 199:145-150.

20. Ng, Y.-S., Wardemann, H., Chelnis, J., Cunningham-Rundles, C., and Meffre, E. 2004. Bruton's tyrosine kinase (Btk) is essential for human B cell tolerance. J. Exp. Med. 200:927-934.

21. Wang, C.Y., Good, R.A., Ammirati, P., Dymbort, G., and Evans, R.L. 1980. Identification of a p69,71 complex expressed on human $T$ cells sharing determinants with B-type chronic lymphocytic leukemic cells. J. Exp. Med. 151:1539-1544.

22. Caligaris-Cappio, F., Gobbi, M., Bofill, M., and Janossy, G. 1982. Infrequent normal B lymphocytes express features of B-cell chronic lymphocytic leukemia. J. Exp. Med. 155:623-628.

23. Kipps, T.J., et al. 1989. Developmentally restricted immunoglobulin heavy chain variable region gene expressed at high frequency in chronic lymphocytic leukemia. Proc. Natl. Acad. Sci. U. S. A. 86:5913-5917.

24. Johnson, T.A., Rassenti, L.Z., and Kipps, T.J. 1997. Ig VH1 genes expressed in B cell chronic lymphocytic leukemia exhibit distinctive molecular features. J. Immunol. 158:235-246.

25. Oppezzo, P., et al. 2004. Somatic mutations can lead to a loss of superantigenic and polyreactive binding. Eur. J. Immunol. 34:1423-1432.

26. Ichiyoshi, Y., and Casali, P. 1994. Analysis of the structural correlates for antibody polyreactivity by multiple reassortments of chimeric human immunoglobulin heavy and light chain $\mathrm{V}$ segments. J. Exp. Med. 180:885-895.

27. Casali, P., Burastero, S.E., Nakamura, M., Inghirami, G., and Notkins, A.L. 1987. Human lymphocytes making rheumatoid factor and antibody to ssDNA belong to Leu-1+ B-cell subset. Science. 236:77-81.

28. Hardy, R.R., Hayakawa, K., Shimizu, M., Yamasaki, K., and Kishimoto, T. 1987. Rheumatoid factor secretion from human Leu-1+ B cells. Science. 236:81-83.

29. Meffre, E., et al. 2000. Circulating human B cells that express surrogate light chains and edited receptors. Nat. Immunol. 1:207-213.

30. Meffre, E., Chiorazzi, M., and Nussenzweig, M.C. 2001. Circulating human B cells that express surrogate light chains display a unique antibody repertoire. J. Immunol. 167:2151-2156.

31. Widhopf, G.F., II, and Kipps, T.J. 2001. Normal B cells express 51p1-encoded Ig heavy chains that are distinct from those expressed by chronic lymphocytic leukemia B cells. J. Immunol. 166:95-102.

32. Chiorazzi, N., Rai, K.R., and Ferrarini, M. 2005. Chronic lymphocytic leukemia. N. Engl. J. Med. 352:804-815.

33. Hayakawa, K., and Hardy, R.R. 2000. Development and function of B-1 cells. Curr. Opin. Immunol. 12:346-353.

34. Martin, F., and Kearney, J.F. 2002. Marginal-zone B cells. Nat. Rev. Immunol. 2:323-335.

35. Weller, S., et al. 2004. Human blood IgM "memory" $B$ cells are circulating splenic marginal zone B cells harboring a prediversified immunoglobulin repertoire. Blood. 104:3647-3654

36. Zupo, S., et al. 1996. CD38 expression distinguishes two groups of B-cell chronic lymphocytic leukemias with different responses to anti-IgM antibodies and propensity to apoptosis. Blood. 88:1365-1374.

37. Chen, L., et al. 2002. Expression of ZAP-70 is associated with increased B-cell receptor signaling in chronic lymphocytic leukemia. Blood. 100:4609-4614.

38. Chen, L., et al. 2005. ZAP-70 directly enhances IgM signaling in chronic lymphocytic leukemia. Blood. 105:2036-2041.

39. Lanham, S., et al. 2003. Differential signaling via surface IgM is associated with $\mathrm{VH}$ gene mutational status and CD38 expression in chronic lymphocytic leukemia. Blood. 101:1087-1093.

40. Damle, R.N., et al. 2004. Telomere length and telomerase activity delineate distinctive replicative features of the B-CLL subgroups defined by immunoglobulin V gene mutations. Blood. 103:375-382.

41. Messmer, B.T., et al. 2005. In vivo measurements document the dynamic cellular kinetics of chronic lymphocytic leukemia B cells. J. Clin. Invest. 115:755-764. doi:10.1172/JCI200523409. 